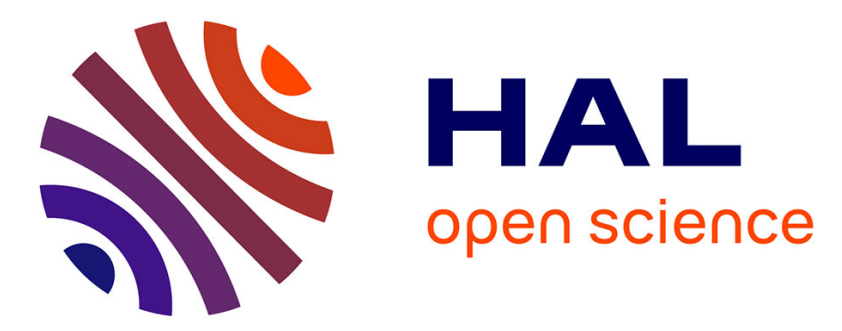

\title{
Multi-resolution analysis techniques and nonlinear PCA for hybrid pansharpening applications
}

\author{
Giorgio Licciardi, Gemine Vivone, Mauro Dalla Mura, Rocco Restaino, \\ Jocelyn Chanussot
}

\section{- To cite this version:}

Giorgio Licciardi, Gemine Vivone, Mauro Dalla Mura, Rocco Restaino, Jocelyn Chanussot. Multiresolution analysis techniques and nonlinear PCA for hybrid pansharpening applications. Multidimensional Systems and Signal Processing, 2015, 27 (4), pp.807-830. 10.1007/s11045-015-0359-y . hal-01259779

\section{HAL Id: hal-01259779 \\ https://hal.science/hal-01259779}

Submitted on 20 Jan 2016

HAL is a multi-disciplinary open access archive for the deposit and dissemination of scientific research documents, whether they are published or not. The documents may come from teaching and research institutions in France or abroad, or from public or private research centers.
L'archive ouverte pluridisciplinaire HAL, est destinée au dépôt et à la diffusion de documents scientifiques de niveau recherche, publiés ou non, émanant des établissements d'enseignement et de recherche français ou étrangers, des laboratoires publics ou privés. 


\title{
Multi-Resolution Analysis Techniques and Nonlinear PCA for hybrid Pansharpening Applications
}

\author{
Giorgio Licciardi • Gemine Vivone • \\ Mauro Dalla Mura • Rocco Restaino • \\ Jocelyn Chanussot
}

Received: date / Accepted: date

\begin{abstract}
Hyperspectral images have a higher spectral resolution (i.e., a larger number of bands covering the electromagnetic spectrum), but a lower spatial resolution with respect to multispectral or panchromatic acquisitions. For increasing the capabilities of the data in terms of utilization and interpretation, hyperspectral images having both high spectral and spatial resolution are desired. This can be achieved by combining the hyperspectral image with a high spatial resolution panchromatic image. These techniques are generally known as pansharpening and can be divided into component substitution (CS) and multi-resolution analysis (MRA) based methods. In general, the CS methods result in fused images having high spatial quality but the fused images suffer from spectral distortions. On the other hand, images obtained using MRA techniques are not as sharp as CS methods but they are spectrally consistent. Both substitution and filtering approaches are considered adequate when applied to multispectral and PAN images, but have many drawbacks when the low-resolution image is a hyperspectral image. Thus, one of the main challenges in hyperspectral pansharpening is to improve the spatial resolution while preserving as much as possible of the original spectral information.

An effective solution to these problems has been found in the use of hybrid approaches, combining the better spatial information of CS and the more accurate spectral information of MRA techniques. In general, in a hybrid approach a CS technique is used to project the original data into a low dimensionality space. Thus, the PAN image is fused with one or more features by means of MRA approach. Finally the inverse projection is used to obtain the enhanced image in the original data space. These methods, permit to effectively enhance the spatial resolution of the hyperspectral image without relevant spectral dis-
\end{abstract}

Giorgio Licciardi, Mauro Dalla Mura and Jocelyn Chanussot

GIPSA-Lab, Grenoble Institute of Technology

E-mail: Giorgio-Antonino.Licciardi@gipsa-lab.grenoble-inp.fr

Gemine Vivone and Rocco Restaino

Universitá di Salerno 
tortions and on the same time to reduce the computational load of the entire process. In particular, in this paper we focus our attention on the use of Nonlinear Principal Component Analysis (NLPCA) for the projection of the image into a low dimensionality feature space. However, if on one hand the NLPCA has been proved to better represent the intrinsic information of hyperspectral images in the feature space, on the other hand, an analysis of the impact of different fusion techniques applied to the nonlinear principal components in order to define the optimal framework for the hybrid pansharpening has not been carried out yet. More in particular, in this paper we analyze the overall impact of several widely used MRA pansharpening algorithms applied in the nonlinear feature space. The results obtained on both synthetic and real data demonstrate that, an accurate selection of the pansharpening method can lead to an effective improvement of the enhanced hyperspectral image in terms of spectral quality and spatial consistency, as well as a strong reduction in the computational time.

Keywords Nonlinear PCA · Hyperspectral image · hybrid pansharpening · Image fusion

\section{Introduction}

A large literature exist on pansharpening and in the last years the number of contributions has grown significantly. The great interest of the community on this topic is proved by dedicated sessions in the most important Remote Sensing and Earth Observation conferences, as well as by the launch of public contests, as the one sponsored by the Data Fusion Committee of the IEEE Geoscience and Remote Sensing Community in 2006 [1]. A taxonomy of pansharpening methods can be found in the literature [2][3]. They can be mainly divided in two groups: the MultiResolution Analysis (MRA) based, and the Component Substitution (CS) methods.

In the MRA framework each image is decomposed, by iterative applications of a given operator, into a sequence of signals (or pyramid) with decreasingly informative content. Moreover, MRA methods can also be divided according to the transformations used for the detail extraction and their injection. The use of pyramidal decomposition as an efficient image representation tool was proposed in [4] by performing a decomposition of the original image via successive lowpass Gaussian filterings and decimations (Laplacian pyramid). The suitability of this method for pansharpening has been shown in [5] especially when an adequate knowledge of the sensor Modulation Transfer Function (MTF) is available [6]. One widely investigated MRA tool relies on the wavelet/contourlet decomposition [7], [8] and yields to notable performances [1]. Both decimated Mallat's method [9], [10] and undecimated approaches (realized through the "à trous" method [11]) have been employed to perform the Discrete Wavelet Transform (DWT). The latter can be easily matched to the sensor MTF [6], but, in principle, the non-orthogonality could compromise the spectral quality of the image [12]. 
In the CS techniques, the low resolution image is projected into a feature space, where one of the obtained features is substituted with the PAN image. The enhanced image is then obtained by reprojecting the features back into the data space. This approach is well represented by the Intensity-Hue-Saturation (IHS) and the Principal Component Analysis methods. The IHS transformation has the ability to separate effectively spatial information (the so-called Intensity component) and spectral information (the Hue and the Saturation components). The fused product is obtained by substitution (after histogram matching) of the panchromatic image with the Intensity component [13]. The application of this method is clearly limited to MS images with only three bands but an extension to any multispectral image has been proposed by $\mathrm{Tu}$ et al. [14]. The PCA [15][3], which is widely used in signal processing, statistics and many other domains, transform the original MS/HS bands into a new set of spectral uncorrelated components. The amount of the data variance is the greatest in the first component, and decreases in the following ones. In general, the first principal component collects the information that is common to all bands used as input data to the PCA, i.e., the spatial information, while the spectral information that is specific to each band is captured in the other principal components. This makes the principal component substitution an adequate technique when merging MS and PAN images. It is worth to underline that, both in the case of IHS and PCA, some spatial information may not be mapped to the first component, depending on the degree of correlation and spectral contrast existing among the MS bands.

In general, the CS methods result in fused images having high spatial quality but with possible spectral distortions [16]. The images fused using methods based on the MRA approach are not usually as sharp as the ones obtained CS but they are spectrally consistent [16]. Moreover, the application of pansharpening techniques designed for multispectral images to hyperspectral images can lead to an increase of spectral distortion. This can be explained with the following example. Let us consider an image with two objects in the scene, one colored in red and one in blue. In a panchromatic image, both objects will differ only in terms of intensity, while in a multispectral image, the two objects will be represented separately in two different bands. This means that inserting spatial information from the panchromatic image into the red band will result in an enhanced red band that have also spatial information from the blue. From this point of view, considering that in a hyperspectral image the number of different spectral bands is higher than in a MS image, it is possible that the injection of the same spatial details in each hyperspectral band may lead to an increase of the spectral distortion to unacceptable values.

Another relevant issue concerning the pansharpening of hyperspectral data is related to the high number of bands that characterizes this imagery. In fact, the application of a pan-sharpening technique to hundreds of bands may result in a not sustainable increase of the computational load and complexity. For this reason a dimensionality reduction preprocessing technique is desired to reduce the computational complexity of the complete process. In the literature, the most used techniques for dimensionality reduction are based on linear trans- 
formations, such as Principal Component Analysis (PCA), Minimum Noise Fraction (MNF) or Independent Component Analysis (ICA). In each of these techniques, the obtained components are ranked in order of relevant information content (cumulative variance, noise, etc...). For instance, in the PCA transformation, most of the original information is projected in the first few components while the remaining ones represent only uncorrelated noise. The dimensionality reduction is then performed by discarding these last components. Based on this assumption, by discarding the last components will then result only in noise removal. However, this approach is effective based on the assumption that in the original data the relevant information is mainly characterized by linear correlations. Unfortunately, many hyperspectral images are not only characterized by linear correlations, consequently, a linear transformation is not able to completely project all the relevant information into the first few components, but part of it could be found also in the other components. A dimensionality reduction performed by discarding the last components of a linear transformation may result in a loss of information and consequently in a spectral distortion as a result of the inverse transformation. Moreover, the first PCA component usually retains information from all the original spectral bands. This means that it may include objects not visible in the spectrum of the PAN image and the fusion process will lead to a further spectral distortion. Therefore a nonlinear approach able to preserve the spectral information of the original image and at the same time reduce the computational load of the process is advisable.

A possible solution to overcome mutual limitations of both CS and MRA approaches, is to use hybrid approaches, combining the different classes of methods in order to find an appropriate balance between spectral and spatial preservation. In the general framework of the hybrid pansharpening, the HS image is firstly projected into a feature space by means of a linear or nonlinear transform, as in CS. Then, according to a MRA approach, spatial filters are applied to the PAN image in order to extract spatial details that will be injected in one or more features obtained in the previous step. Finally an inverse transform is applied in order to obtain the enhanced image. In this way it is possible to reduce the spectral distortion produced by the substitution process. On the other hand, the spatial enhanced image may present less spatial details than the image obtained using a classic CS technique. Thus, depending on the selected filtering method, the final image may result in an optimal tradeoff between spectral and spatial quality. Few examples of hybrid pansharpening approaches have been proposed in the literature. A hybrid approach has been proposed in [5], where GS and gaussian filters have been combined to enhance the quality of MS images. An alternative solution has been proposed in [17] exploiting the synergic combination of NonLinear Principal Component Analysis (NLPCA) [18] and INDUSION [19]. In particular, the NLPCA has been used to project the low resolution HS image into a reduced dimensionality feature space. Then, the spatial information from the high resolution PAN image has been injected into the obtained nonlinear principal components (NLPCs) by means of INDUSION. Finally, the enhanced features have been reprojected 
back to the original data space using the inverse NLPCA function in order to obtain the enhanced HS image. The obtained results demonstrated that the use of NLPCA can effectively improve the quality of the enhanced hyperspectral if compared with the results obtained applying the INDUSION directly to the original image. While it has been demonstrated that, compared to other similar approaches, the NLPCA is able to retain more relevant information in less components [20], an in deep analysis of the most suitable MRA techniques for the pansharpening of NLPCs is still missing. Moreover, in [21] it has been pointed out that the NLPCs may be more related to different parts of the spectrum, thus suggesting the fusion of the PAN image with only a subset of NLPCs. For these reasons in this paper we propose an extensive analysis in terms of pansharpening technique as well as the method to select the features to be used.

The remainder of this paper is the following. Nonlinear transformations and the NLPCA method, are is described in Section 2, while Section 3 reports the validity of the proposed technique in two test cases: The first one is a synthetic dataset obtained from by the ROSIS sensor. In the second case a real HS image acquired by the CHRIS-Proba sensor will be enhanced by using the spatial information coming from a QuickBird Panchromatic image. Finally, the conclusions and perspectives are given in Section 4.

\section{NLPCA-based hybrid pansharpening method}

In the literature many methods have been proposed to extract component in a nonlinear manner, e.g., Locally linear embedding (LLE) [22] and Isomap [23] visualize high dimensional data by projecting (embedding) them into a two or three-dimensional space. Principal curves and self organizing maps (SOM) [24] describe data by nonlinear curves and nonlinear planes up to two dimensions. The main limitation of these methods is related to obtaining low number of features, that may be not sufficient to describe the inherent information of the data. An alternative solution to NLPCA can be offered by the Kernel Principal Components Analysis (KPCA) [25]. In KPCA the original data is firstly mapped into a higher dimensional feature space $F$, and then PCA is performed in $F$ to extract nonlinear principal components of the input data. Due to the high computational complexity, the mapping into a higher feature space can be exploited by applying the kernel trick method. The kernel trick in machine learning is a way to easily adapt linear algorithms to nonlinear situations. In the case of KPCA, the kernel trick permits to projects the input data into a higher dimensional implicit feature space $F$ without having to compute the mapping explicitly. Similarly to PCA, the dimensionality reduction is performed by discarding the less relevant components. Both KPCA and NLPCA methods could be considered as a nonlinear generalization of the standard PCA and tend to produce similar results in terms of feature space. However, being the feature space $F$ implicit and unknown, is not always possible to find the exact demapping function from $\mathrm{F}$ to the original data space. 
The reconstructed data can be obtained by minimizing the reconstruction error in $F$ with gradient descent method. Thus, the results obtained with this approach are quite far form the optimal solution, presenting high amount of spectral distortion. For these reasons in this paper we analyze the NonLinear Principal Component Analysis and its application to the hybrid pansharpening framework.

\subsection{NonLinear Principal Component Analysis}

Let us consider a set of $n$ observations of $m$ dimensionality, denoted as $\mathbf{X}=$ $\left[\mathbf{x}_{1}, \ldots, \mathbf{x}_{i}, \ldots, \mathbf{x}_{m}\right]$ with $\mathbf{x}_{i}$ the column vector correspondent to the $i$-th variable for the $n$ observations. In NLPCA, the mapping into a feature space of lower dimensionality is performed through arbitrary nonlinear functions in the form:

$$
\mathbf{Y}=\mathbf{g}(\mathbf{X}) \text {, }
$$

where the $n \times f$ resulting matrix $\mathbf{Y}=\left[\mathbf{y}_{1}, \ldots, \mathbf{y}_{j}, \ldots, \mathbf{y}_{f}\right]$ represents the set of the $f<m$ NonLinear Principal Components (NLPCs) and $\mathbf{g}=\left[g_{1}, \ldots, g_{f}\right]$ is the set of $f$ nonlinear functions. The $j$-th NLPC is obtained by applying the $j$-th mapping to $\mathbf{X}$, i.e.:

$$
\mathbf{y}_{j}=g_{j}(\mathbf{X}), \forall j=1, \ldots, f .
$$

The inverse transformation, which aims at re-mapping the data from $f$ back to the original $m$-dimensionality, can be performed with a set of $m$ nonlinear transformations $\mathbf{h}=\left[h_{1}, \ldots, h_{m}\right]$ :

$$
\widehat{\mathbf{X}}=\mathbf{h}(\mathbf{Y})
$$

where the $n \times m$ matrix $\widehat{\mathbf{X}}$ is the reconstruction of $\mathbf{X}$. The $i$-th reconstructed variable $\widehat{\mathbf{x}}_{i}$ is given by:

$$
\widehat{\mathbf{x}}_{i}=h_{i}(\mathbf{Y}), \forall i=1, \ldots, m
$$

The loss of information between the original and reconstructed data can be measured by computing the error matrix $\mathbf{E}=\mathbf{X}-\widehat{\mathbf{X}}$.

\subsection{AutoAssociative Neural Network}

In [26] it was shown that any nonlinear function can be approximated by a superposition of a set of $\sigma(x)$ transformations that are functions continuous and monotonically increasing, with $\sigma(x) \rightarrow 1$ as $x \rightarrow+\infty$ and $\sigma(x) \rightarrow 0$ as $x \rightarrow-\infty$. This property is often called universal fitting. Sigmoidal functions are an example of transformations fulfilling these constraints [27]. The approximation of a nonlinear function can be obtained by an Artificial Neural Networks (ANNs) using one layer of nodes with sigmoidal activation function 
and two layers of weighted connections [27]. In practice, sigmoidal nonlinearities are often included in the nodes of the output layer so that the network produces outputs in a fixed and finite range. The ability of the NN to fit arbitrary nonlinear functions depends on the presence of a hidden layer with nonlinear nodes. Without the hidden layer (or with linear nodes in the hidden layer), the network is only capable of producing linear combinations of the inputs, given linear nodes in the output layer. A network lacking a hidden layer but including sigmoidal nonlinearities in the output layer is only capable of generating multivariable sigmoidal functions.

Starting from these considerations, the NLPCA can be implemented by two ANNs approximating the nonlinear functions $\mathbf{g}$ and $\mathbf{h}$, as depicted in Fig. 1. The ANN approximating $\mathbf{g}$, called coding subnet, has as an input layer $m$ nodes followed by the hidden layer (often called mapping layer) with $M_{1}>f$ nodes and sigmoidal transfer functions (to verify the universal fitting property). The output layer of this subnet contains $f<m$ nodes and for this reason it is often called bottleneck. The second ANN (also called decoding subnet) approximates the $\mathbf{h}$ function. Its input layer has $f$ nodes followed by the hidden layer (often called demapping layer) with $M_{2}>f$ nodes and sigmoidal transfer functions (to verify the universal fitting property). The output layer yields the reconstructed data and thus contains $m$ nodes. The nodes of the output layers can be linear or sigmoidal. ANNs require supervised training which translates in tuning the network in order to obtain the desired output for each training example. However, the outputs of the coding subnet, and hence the inputs of the following decoding subnet, are unknown. Conversely, the input and the outputs of the coding and decoding networks, respectively are known. Therefore, direct supervised training of these networks is not feasible. To circumvent this problem, one can observe that combining in series the two ANNs or equivalently defining a composite function $\mathbf{z}(\cdot)=(\mathbf{h} \circ \mathbf{g})(\cdot)=\mathbf{h}(\mathbf{g}(\cdot))$ that links the original data $\mathbf{X}$ with their reconstruction version $\widehat{\mathbf{X}}$ :

$$
\widehat{\mathbf{X}}=\mathbf{z}(\mathbf{X})
$$

the combined network can be trained to produce the identity mapping. This means that the parameters of the network representing $\mathbf{z}$ are optimized so that the reconstructed outputs match the inputs as closely as possible. The training aimed at learning the identity mapping has been called self-supervised backpropagation or autoassociation [28] leading to the definition of AutoAssociative NNs (AANNs). For AANNs the training phase is an iterative process and is completed when the sum of squared errors is minimized:

$$
E=\sum_{p=1}^{n} \sum_{i=1}^{m}\left(\widehat{\mathbf{x}}_{i}-\mathbf{x}_{i}\right)_{p}{ }^{2}
$$

or equivalently when the matrix error norm $\|\mathbf{E}\|$ is minimized (as for the case of the PCA).

Once the complete AANN $\mathbf{z}$ is trained, it is possible to use the coding subnet to project the original data into a lower dimensional space. Thus, the 
$f$ NLPCs can be obtained from the output layer of the coding subnet, which is also called the bottleneck. The obtained NLPCs can be subsequently used as input to the decoding subnet in order to obtain the reconstructed original data.

As it can be seen, the main difference between PCA and NLPCA is that the latter is able to map nonlinear relations between variables, while PCA is only able to deal with linear ones. This means that, NLPCA has the relevant advantage to retain most of the variance of the data in fewer components with respect to PCA.

One of the main difficulties in designing the AANN relies in the selection of the correct number of nodes that minimizes the loss of information produced in the three hidden layers, and in particular in the bottleneck layer. Being the AANN designed in order to minimize the reconstruction error, the best NN topology can be retrieved by using a simple grid search algorithm that varies recursively the number of nodes of the hidden layers and evaluated the respective error. Then, the topology presenting the lowest error is selected [17]. However, without a starting point, this approach can be extremely time consuming and a different solution should be found. Starting from $n$ that represents the number of samples in the training set, a separate constraint is imposed by each output node, so that the total number of the possible adjustable parameters (weights and biases for all network connections and nodes, respectively) must be less than $n \cdot m$. Moreover, analyzing the structure of the AANN used here, it can be found that the number of adjustable parameters is $\left(M_{1}+M_{2}\right)(m+f+1)+m+f$ that implies the following inequality:

$$
M_{1}+M_{2} \ll \frac{m(n-1)-f}{m+f+1} .
$$

The aim of a dimensionality reduction method is to reduce the original spectral dimension into a lower dimensional space. This can be translated into the AANN structure as a condition on $f$, i.e. $f \ll m, n$. Then, eq. (7) becomes:

$$
M_{1}+M_{2} \ll n
$$

Assuming a balanced structure of the AANN, $M_{1}$ and $M_{2}$ should have the same dimensions $\left(M_{1}=M_{2}=M\right)$, we have:

$$
2 M \ll n .
$$

It is worth to note that eq. (9) is effective only if the number of mapping/demapping nodes $M$ is greater then the number of nodes in the bottleneck layer $f$. Otherwise, there will not be enough data to effectively extract $f$ NLPCs. It has to be noted that, since the output has to simply replicate the input, there is no need to have an a priori knowledge for the implementation of the learning phase. This implies that the AANN training can be performed in a fully automatic way and that all pixels in the image can be considered for this task, which has actually been the technique adopted in this paper.

To reduce the distortions and enhance the spatial information we propose a 
hybrid approach that combines both the advantages of substitution and filtering based methods. In particular, it has been noted that, similarly to the classical PCA approach, each NLPC seems to be polarized to different types in the scene. In particular, in [29] it has been demonstrated that the nonlinear components retain information about different parts of the spectrum. For this reason we propose to enhance spatially not all the nonlinear components, but only to those components presenting most similarities with the PAN image.

2.3 Proposed pansharpening technique based on NLPCA

Entering in more detail, we call the hyperspectral low spatial resolution image HSLR. Let us define the vectorized form of the panchromatic image $\mathbf{P}$ as $\mathbf{p}=\operatorname{vec}(\mathbf{P})$, where vec is the operator that transforms the original image in its vectorized form (i.e., concatenating the pixels of the image column- or rowwise). Then the interpolated version of HSLR (in order to have the same pixel number of $\mathbf{p}$ for each band) is defined as $\widetilde{\mathbf{H S L R}}=\left[\widetilde{\mathbf{h s l r}}_{1}, \ldots, \widetilde{\mathbf{h s l r}} \boldsymbol{i}, \ldots, \widetilde{\mathbf{h s l r}}{ }_{B}\right]$ where $\widehat{\mathbf{h s l r}}_{i}$ is the vectorized form of the $i$-th band of the interpolated hyperspectral image and $B$ represents the band number. The first step of the approach is to train the AANN using the whole data set $\widetilde{\text { HSLR }}$ in order to obtain the functions $\mathbf{g}$ and $\mathbf{h}$ for the coding and decoding phases, respectively. Then, we can exploit eq. (1) to get the set of $f$ NLPCs called $\mathbf{N L P C}=\left[\mathbf{n l p c}_{1}, \ldots, \mathbf{n l p c} \mathbf{c}_{f}\right]$ from now on. In eq. (1), the role of $\mathbf{X}$ is played by $\widetilde{\mathbf{H S L R}}$.

Since the NLPCs tends to be represent different characteristics of the spectra, the injection of the spatial details of the PAN indiscriminately into each component would not lead to an acceptable result. To solve this problem it has been decided to select the NLPCs in which inject the spatial details by evaluating the correlation with the PAN image. If the result is higher than a given threshold $\theta$, the fusion between the specific high correlated NLPC and the panchromatic image is done, otherwise the original NLPC is held. In particular, a MultiResolution Analysis (MRA) fusion approach is exploited to reduce the spectral distortion w.r.t. the classical substitution of the NLPC with a histogram matched version of the panchromatic image. Therefore, only the details of the histogram matched panchromatic image are injected into the NLPC to get the fusion product. Several fusion approaches have been tested in this paper and these will be described in Sec.3.2. At the end, the subset of NLPCs fused with the panchromatic image and the subset of NLPCs which did not exceed the threshold for the fusion are used in eq. (3) to obtain the final fused product $\mathbf{H S H} \mathbf{R}$. The complete processing proposed in this paper is resumed in Algorithm 1 and Fig. 2. 


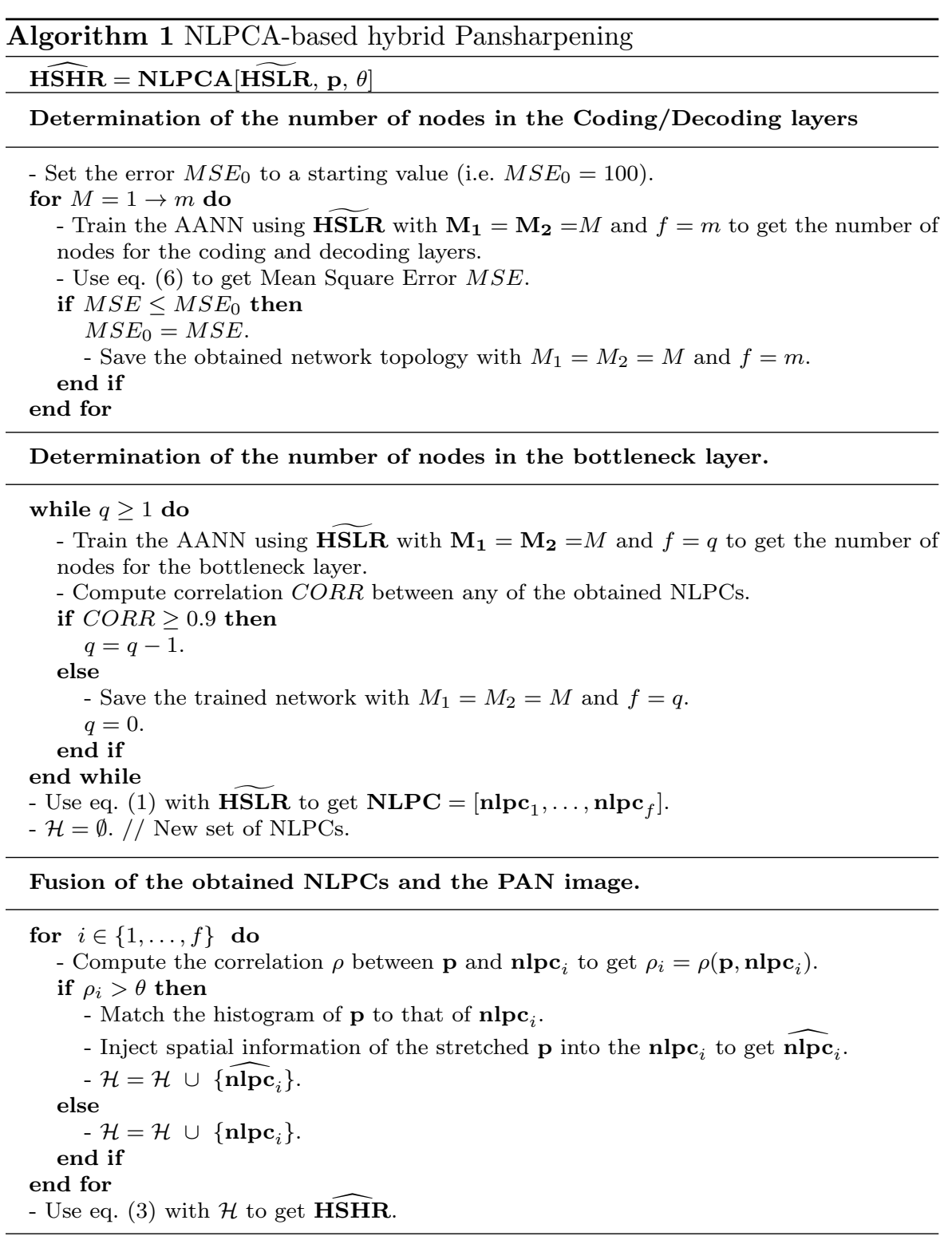

\section{NLPCA hybrid configuration analysis}

Scope of this paper is to retrieve the optimal configuration for the proposed NLPCA hybrid approach for the pansharpening of hyperspectral images. In particular, in this section we analyze the impact of several well known MRA techniques applied in the nonlinear feature space, on the spatial and spectral qualities of final enhanced hyperspectral images. In order to retrieve the opti- 
mal configuration we tested the proposed hybrid framework using the following MRA methods:

- CBD, Context-Based Decision and the approach proposed in[5] using $\vartheta=$ 0.3 and [9X9] window size.

- ATWT, A Trous Wavelet with additive injection model [11]

- HPF, Box window and HPF injection model [3]

- MF, Morphological Filter and High Pass Modulation (HPM) injection model [30]

- GLP, Gaussian filter with MTF adjustment and HPF injection model [6]

- MTF-HPM, Gaussian filter with MTF adjustment and HPM injection model [6][31]

- AWLP, Additive Wavelet Luminance Proportional [32]

- SFIM, Smoothing filter-based intensity modulation [33]

It worth to be noticed that the Indusion technique [19], used in [17], can be considered as a special case of the AWRGB technique, so it was not considered in this evaluation.

A further analysis has been carried out by comparing the results obtained following the hybrid approach with those obtained applying the MRA techniques directly to the HS images. The following CS methods were also included in the analysis:

- NLPCA method based on the substitution of one or more NLPCs with the PAN image [29]

- PCA method based on the substitution of the first PCs with the PAN image [15]

The above mentioned approaches have been applied to two hyperspectral images, presenting different characteristics. For each test an enhancement ratio of 4 has been selected because is a typical value that offers the best tradeoff between spatial enhancement and spectral distortion [19].

The quantitative analysis of the quality of the enhanced images has been performed through the use of Spectral Angle Mapper (SAM), Relative Global Error of Synthesis (ERGAS) and spatial correlation coefficient (SCC) [3] [34]. While SAM is useful for measuring the spectral quality of the fusion process, ERGAS can measure both spectral and spatial quality. The ideal value for SAM and ERGAS indexes, meaning no distortion between the pansharpened and reference image, is zero. However, considering fused images, values that are around 3 are referred to a good image enhancement. The SCC index measures the spatial correlation between two images. Ideal value for the merged image is 1 , indicating that the whole spatial information of one of the images is present in the other one. 


\subsection{ROSIS-University dataset}

In the first experiment we tested a synthetic dataset derived from the University dataset [35]. The Synthetic hyperspectral image is obtained reducing the spatial resolution of a 103 bands hyperspectral image acquired by the ROSIS airborne sensor, while the panchromatic image is obtained by averaging the values of the ROSIS bands. This choice have the relevant advantage that the resulting pansharpened image does not suffer from distortion caused by misregistration between the HS and PAN. Moreover, both synthetic PAN and HS images have the same spectral coverage and temporal coherence, avoiding the insurgence of further spectral distortions. Another important issue related with this data is the correlation between the different spectral bands that is mainly linear. This should suggest us that based on the assumption that the NLPCA approach is able to deal with both linear and nonlinear correlations, the results obtained using component substitution methods on the features obtained with PCA and NLPCA should be similar.

According to the procedure proposed by Wald [36], both the synthetic HS image has been degraded to a lower resolution with a ratio of 4 , by means of bicubic convolution downscaling. This is done so that the resultant pansharpened image is at the same resolution as the starting reference (i.e. the original HS image), and hence statistical analysis can be made between the reference and the pansharpened images.

According to the proposed method, the first step is to project the HS image into a nonlinear feature space by means of NLPCA. The Pavia dataset features 103 bands, that correspond to 103 nodes in the input/output layers. Following the procedure described in the previous section, a grid search to optimize the number of nodes in the three hidden layers of the nonlinear AANN has been carried out. The best topology has been found to have 50 nodes both in the coding/decoding layers and 4 nodes in the bottleneck layer,that correspond to 4 NLPCs. In order to preserve as much as possible the spectral information of the HS image while enhancing the spatial resolution, it could be possible to perform the pansharpening only on a subset of components. This can be exploited by comparing the PAN image with the 4 NLPCs in order to detect the most similar component. As can be noted from from Fig. 3, from a qualitative point of view, component 3 presents characteristics very similar to the PAN image. Also analyzing the correlation values between components and PAN image, it can be noted that component 3 reaches the highest correlation, as reported in Table 1 . These values would suggest that components 2 and 3 presents similar characteristics with the PAN image. However, also component 4 presents a certain amount of similarity with the PAN. In order to detect the best subset of components, in this experiments four different configurations have been tested combining the PAN image with component 3, components 2 and 3, components 2, 3 and 4 and all the components, respectively. In each test, the selected components have been then spatially enhanced and substituted in the NLPC dataset in place of the original ones. The new feature datasets have been then reprojected back to the original spectral domain using the decoding 
subnet of the AANN.

Tables 2-10 report the ERGAS, SAM and SCC quality indexes associated to the different pansharpening techniques. As expected, among the results obtained with the proposed approach, the best tradeoff between spectral and spatial quality is obtained by fusing the PAN image with components 2 and 3 , suggesting that using only component 3 is not sufficient even if it is the most correlated with the PAN image. Moreover, components 4 and 1, don't improve the quality of the enhanced images, but in most cases tend to increase the distortions. This suggests that a threshold on the correlation between PAN and components should be defined. On a comparison of these results with those obtained applying the same fusion techniques directly to the spectral bands it can be noted that use of the NLPCA preprocessing improves the spectral quality of the enhanced images preserving the spatial coherency.

As expected, comparing the results obtained using the linear (PCA) and the nonlinear PCA (SUB), the two methods permit to produce enhanced images that have approximately the same spectral quality, evaluated in terms of SAM. However, on the other hand, the use of NLPCA permits to have images with high spatial information, in terms of SCC, than the linear PCA. As for the pansharpening techniques, MTF-HPF, applied to components 2 and 3, achieve the best result in terms of spectral quality, while AWLP applied to the same components presents the higher spatial consistency value. On a more general evaluation, AWRGB, AWLP and MTF-HPF applied to NLPCs 2 and 3 present the best tradeoff between spatial information and spectral distortion.

Also from a qualitative point of view, the enhanced images obtained performing the pansharpening on components 2 and 3 in the nonlinear feature space appear to be sharper and more spectrally consistent than the images obtained by performing the pansharpening in the original data space, as depicted in Fig. 4 and 5.

A further analysis is carried out in order to evaluate the computational loads of the different methods. Table 11 reports the required time, expressed in seconds, for each technique to produce the enhanced image. The computation time analysis has been carried out for each technique on a $2.5 \mathrm{GHz}$ intel Core i7 processor with $16 \mathrm{~GB} 1600 \mathrm{MHz}$ memory. As it can be clearly seen, the use of NLPCA extremely improves the computational efficiency of the fusion process. It has to be noted that these values include also an overall transformation time of 5.0s for this image.

\subsection{CHRIS-Proba dataset}

On a second experiment we used real images acquired under different conditions. In particular the hyperspectral image is a CHRIS mode-3 image acquired by the Proba satellite, while the panchromatic image is acquires from the Quickbird satellite. The CHRIS-Proba image presents 18 bands and a spatial resolution of $20 \mathrm{~m}$, while the PAN image has $1 \mathrm{~m}$ resolution. The two images were acquired in different period of 2006 over the Tor Vergata area, 
southeast of Rome, Italy, presenting differences in terms of spectral coverage, atmospheric condition and illuminations as well as small differences of the cultivated areas. In this case the inter-bands correlation has been proven to be nonlinear [37]. Thus we expect that the use of a nonlinear approach should improve the quality of the enhanced images.

As for the previous experiment, a grid search algorithm has been used to find the optimal number of nodes in the hidden layers of the AANN. The best AANN topology configuration has been found to have 18 inputs/output nodes, 9 nodes both in the outer hidden layers and 3 nodes in the bottleneck layer, corresponding to 3 NLPCs.

Being the two images acquired in different dates, with different atmospheric conditions and with different angles of view, high correlation values between the NLPCs and the PAN image are not expected, as can be clearly seen from Fig. 6 and Table 12. In this case, only one component (NLPC 3) presents an appreciable correlation characteristics with the PAN image. For this reason, only component 3 has been taken into account to be fused with the PAN image. For each pansharpening technique, the selected component has been spatially enhanced and substituted in the NLPC dataset in place of the original one. Then, each enhanced feature dataset has been reprojected back to the original spectral domain using the demapping subnet of the nonlinear AANN.

In this experiment we decided to not apply the Wald procedure because reducing the CHRIS image to a lower resolution there will be no significant information left in the images and hence the pansharpening techniques would return poor results [17]. Since the Wald procedure cannot be applied, the ERGAS index, measuring both spectral and spatial distortions, should not be used since the reference image is at a lower resolution, thus it does not contain both kind of information. For this reason we considered SAM, as spectral quality index, evaluated between the enhanced image and the original HS image, and SCC, evaluated between the enhanced image and the PAN image, for the spatial quality. Even if the PAN image as a reference should not be used as a general evaluation index, it is possible to use it as a term of comparison of the ability of the different techniques to extract spatial details [2]. From Tables 13 and ??, showing the SAM and SCC quality indexes associated to the different approaches, it can be see that in most of the cases, independently from the chosen pansharpening technique, the projection into the nonlinear feature space permits to achieve better results, in terms of spectral and spatial quality, if compared with the same fusion method applied directly to the hyperspectral image. In particular, the enhanced images obtained with a NLPCA preprocessing present good tradeoffs between the SAM and SCC that in the worst cases, is comparable to the direct approach.

As expected, the substitution of NLPC 3 with the PAN image permits to obtain better quality of the enhanced image, if compared to the linear PCA. This is also evident by qualitatively analyzing Fig. 7. In particular, it can be noted that while the image obtained following the PCA approach presents evident spectral distortions, the image obtained substituting NLPC3 with the PAN image is spatially well defined and presents spectral characteristics similar to 
the original image.

As for the pansharpening techniques, in this case, the best spectral quality has been achieved by the CBD method, while the best spatial consistency is obtained by substituting the PAN image to component 3. On a more general analysis, the best tradeoff between spectral and spatial qualities have been achieved by AWRGB, AWLP and MTF-HPF methods.

Table 15 shows the required time, expressed in seconds, for each technique to produce the enhanced image. Also in this case the use of NLPCA improve the computational efficiency of the image enhancement process. The overall time to perform the NLPCA transformation has been measured to be $0.16 \mathrm{~s}$.

\section{Conclusions}

In this paper the synergic use of projection into nonlinear feature space and different pansharpening techniques has been investigated in the framework of image spatial enhancement. In particular, in the proposed approach the hyperspectral image is projected into a low dimensional feature space by means of NLPCA. The obtained nonlinear principal components are then enhanced spatially and reprojected back to the original data space. Several pansharpening techniques have been considered and applied to the components that present the highest correlation values. The proposed approach has been applied to both synthetic and real images, characterized by linear and nonlinear inter-bands correlations, respectively. SAM, SCC and ERGAS indexes have been used to evaluate the enhanced images in terms of spectral distortion and spatial consistency. Qualitative analysis has been carried out by visual inspecting the enhanced images.

Analyzing the values of the quality indexes it has been demonstrated that the use of the NLPCA transformation permits to improve the quality of the enhanced images, with regard to the same pansharpening method applied in the data space. A direct comparison of the substitution methods based on PCA and NLPCA confirms the supposition that the nonlinear method, able to to map both linear and nonlinear relations between variables, permits to NLPCA to project data with greater accuracy in fewer components than PCA, if nonlinear correlations exist between input variables and consequently resulting in better quality of the enhanced images.

As for the different fusion techniques, in both experiments, AWRGB, AWLP and MTF-HPF applied to the NLPCs obtained from the NLPCA transformation obtained the best results in terms of spectral and spatial quality.

\section{Tables}




\begin{tabular}{ccccc}
\hline & NLPC 1 & NLPC 2 & NLPC 3 & NLPC 4 \\
PAN & -0.0536 & 0.9064 & 0.9477 & 0.2870 \\
\hline
\end{tabular}

Table 1 Correlation coefficients between PAN image and the 4 NLPCs obtained from the ROSIS dataset.

\begin{tabular}{lcccc}
\hline NLPCs & 3 & 2,3 & $2,3,4$ & $1,2,3,4$ \\
\hline CBD & 6.6976 & 6.5437 & 7.4711 & 7.3906 \\
ATWT & 6.7157 & 6.5087 & 6.8447 & 6.6953 \\
HPF & 6.8376 & 6.6364 & 6.9274 & 6.8534 \\
MF & 7.2987 & 13.2843 & 14.0679 & 15.1341 \\
GLP & 6.7972 & 6.4943 & 6.8159 & 6.6975 \\
MTF-HPM & 6.7369 & 6.5685 & 6.9588 & 6.7327 \\
AWLP & 6.7157 & 6.5087 & 6.8447 & 6.6957 \\
SFIM & 6.8380 & 6.6978 & 7.0706 & 6.8425 \\
\hline
\end{tabular}

Table 2 SAM quality index values (measured in degrees), obtained using the hybrid approach with different MRA techniques applied to the ROSIS image.

\begin{tabular}{ccccc}
\hline NLPC 3 & NLPCs 2, 3 & NLPCs 2, 3, 4 & NLPCs 1, 2, 3, 4 & PCA \\
\hline 7.0120 & 6.5856 & 9.9686 & 9.5204 & 6.6061 \\
\hline
\end{tabular}

Table 3 SAM quality index values (measured in degrees), obtained with different CS techniques applied to the ROSIS image.

\begin{tabular}{lccccccc}
\hline CBD & ATWT & HPF & MF & GLP & MTF-HPM & AWLP & SFIM \\
\hline 6.8489 & 6.8832 & 6.8021 & 12.1443 & 6.8451 & 8.9873 & 6.7253 & 11.1472 \\
\hline
\end{tabular}

Table 4 SAM quality index values (measured in degrees), obtained with different MRA techniques applied directly to the ROSIS image.

\begin{tabular}{lcccc}
\hline NLPCs & 3 & 2,3 & $2,3,4$ & $1,2,3,4$ \\
\hline CBD & 7.1653 & 7.1475 & 8.4181 & 8.4115 \\
ATWT & 7.1346 & 7.1002 & 7.9359 & 7.8999 \\
HPF & 7.1860 & 7.1711 & 8.0134 & 8.0237 \\
MF & 7.2591 & 10.7230 & 11.0310 & 10.9468 \\
GLP & 7.1406 & 7.0995 & 7.8815 & 7.8650 \\
MTF-HPM & 7.1325 & 7.0783 & 8.4154 & 8.2797 \\
AWLP & 7.1346 & 7.1007 & 7.9359 & 7.8997 \\
SFIM & 7.1459 & 7.1140 & 8.3495 & 8.2264 \\
\hline
\end{tabular}

Table 5 ERGAS quality index values obtained using the hybrid approach with different MRA techniques applied to the ROSIS image.

\begin{tabular}{ccccc}
\hline NLPC 3 & NLPCs 2, 3 & NLPCs 2, 3, 4 & NLPCs $1,2,3,4$ & PCA \\
\hline 7.0540 & 6.9151 & 16.0414 & 15.6749 & 8.8601 \\
\hline
\end{tabular}

Table 6 ERGAS quality index values, obtained with different CS techniques applied to the ROSIS image. 


\begin{tabular}{lccccccc}
\hline CBD & ATWT & HPF & MF & GLP & MTF-HPM & AWLP & SFIM \\
\hline 8.6790 & 6.6527 & 6.0725 & 7.1877 & 6.5513 & 7.2173 & 6.7529 & 7.3360 \\
\hline
\end{tabular}

Table 7 ERGAS quality index values, obtained with different MRA techniques applied to the ROSIS image.

\begin{tabular}{lcccc}
\hline NLPCs & 3 & 2,3 & $2,3,4$ & $1,2,3,4$ \\
\hline CBD & 0.7984 & 0.8144 & 0.7851 & 0.7880 \\
ATWT & 0.7985 & 0.8199 & 0.7989 & 0.8013 \\
HPF & 0.7911 & 0.8104 & 0.7876 & 0.7859 \\
MF & 0.7514 & 0.7558 & 0.6890 & 0.6665 \\
GLP & 0.7972 & 0.8178 & 0.7982 & 0.7978 \\
MTF-HPM & 0.7964 & 0.8140 & 0.8031 & 0.8056 \\
AWLP & 0.7985 & 0.8765 & 0.7989 & 0.8012 \\
SFIM & 0.7910 & 0.8061 & 0.7898 & 0.7903 \\
\hline
\end{tabular}

Table 8 SCC quality index values obtained using the hybrid approach with different MRA techniques applied to the ROSIS image.

\begin{tabular}{ccccc}
\hline NLPC 3 & NLPCs 2, 3 & NLPCs 2, 3, 4 & NLPCs 1, 2, 3, 4 & PCA \\
\hline 0.8364 & 0.8669 & 0.7565 & 0.7288 & 0.7737 \\
\hline
\end{tabular}

Table 9 SCC quality index values obtained with different CS techniques applied to the ROSIS image.

\begin{tabular}{lccccccc}
\hline CBD & ATWT & HPF & MF & GLP & MTF-HPM & AWLP & SFIM \\
\hline 0.8196 & 0.8625 & 0.8467 & 0.6953 & 0.8620 & 0.7926 & 0.8797 & 0.6928 \\
\hline
\end{tabular}

Table 10 SCC quality index values, obtained with different MRA techniques applied to the ROSIS image.

\begin{tabular}{lccccc}
\hline NLPCs & 3 & 2,3 & $2,3,4$ & $1,2,3,4$ & Original \\
\hline CBD & 6.25 & 7.16 & 7.97 & 9.66 & 80.48 \\
ATWT & 5.29 & 5.55 & 5.80 & 6.28 & 23.81 \\
HPF & 5.10 & 5.14 & 5.17 & 5.25 & 2.43 \\
MF & 5.26 & 5.36 & 5.49 & 5.64 & 8.86 \\
GLP & 6.33 & 7.37 & 8.86 & 11.59 & 104.99 \\
MTF-HPM & 6.15 & 5.37 & 8.51 & 11.33 & 104.75 \\
AWLP & 5.29 & 5.56 & 5.82 & 6.33 & 32.06 \\
SFIM & 5.07 & 5.10 & 5.18 & 5.21 & 2.45 \\
NLPCA & 5.11 & 5.15 & 5.15 & 5.20 & $/$ \\
PCA & $/$ & $/$ & $/$ & $/$ & 8.55 \\
\hline
\end{tabular}

Table 11 Computational time (expressed in seconds) evaluated for the different methods applied to the ROSIS image.

\begin{tabular}{cccc}
\hline & NLPC 1 & NLPC 2 & NLPC 3 \\
PAN & -0.0906 & 0.2340 & 0.3951 \\
\hline
\end{tabular}

Table 12 Correlation coefficients between PAN image and the 3 NLPCs obtained from the CHRIS dataset. 


\begin{tabular}{lcc}
\hline & SAM & SCC \\
\hline CBD & 2.9546 & 0.4678 \\
ATWT & 3.1674 & 0.8029 \\
HPF & 3.1074 & 0.7241 \\
MF & 3.3483 & 0.6878 \\
GLP & 3.1597 & 0.7973 \\
MTF-HPM & 3.2477 & 0.7817 \\
AWLP & 3.1674 & 0.8029 \\
SFIM & 3.2007 & 0.7019 \\
\hline
\end{tabular}

Table 13 SAM (expressed in degrees) and SCC quality indexes values obtained using the hybrid approach with different MRA techniques applied to the CHRIS image.

\begin{tabular}{|c|c|c|}
\hline \multicolumn{3}{|c|}{ MRA techniques } \\
\hline & SAM & $\mathrm{SCC}$ \\
\hline CBD & 4.2131 & 0.4767 \\
\hline ATWT & 4.0330 & 0.8615 \\
\hline $\mathrm{HPF}$ & 3.5904 & 0.7869 \\
\hline $\mathrm{MF}$ & 4.4187 & 0.2074 \\
\hline GLP & 3.9313 & 0.8562 \\
\hline MTF-HPM & 3.0438 & 0.1929 \\
\hline AWLP & 5.6832 & 0.7924 \\
\hline SFIM & 2.9517 & 0.3083 \\
\hline \multicolumn{3}{|c|}{ CS techniques } \\
\hline & SAM & SCC \\
\hline NLPCA & 3.6093 & 0.8893 \\
\hline PCA & 9.9237 & 0.6624 \\
\hline
\end{tabular}

Table 14 SAM (expressed in degrees) and SCC quality indexes values obtained with different MRA and CS techniques applied directly to the CHRIS image.

\begin{tabular}{lcc}
\hline & hybrid-NLPCA & original \\
\hline NLPCA & 0.16 & $/$ \\
CBD & 1.00 & 24.45 \\
ATWT & 0.36 & 6.81 \\
HPF & 0.23 & 2.69 \\
MF & 0.37 & 4.29 \\
GLP & 1.21 & 45.26 \\
MTF-HPM & 0.95 & 27.03 \\
AWLP & 0.37 & 5.40 \\
SFIM & 0.21 & 1.79 \\
PCA & $/$ & 0.84 \\
\hline
\end{tabular}

Table 15 Computational time (expressed in seconds) evaluated for the different methods applied to the CHRIS image.

\section{Figures}




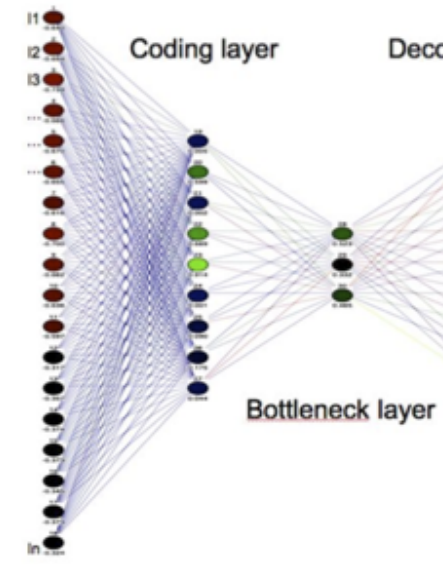

Input layer

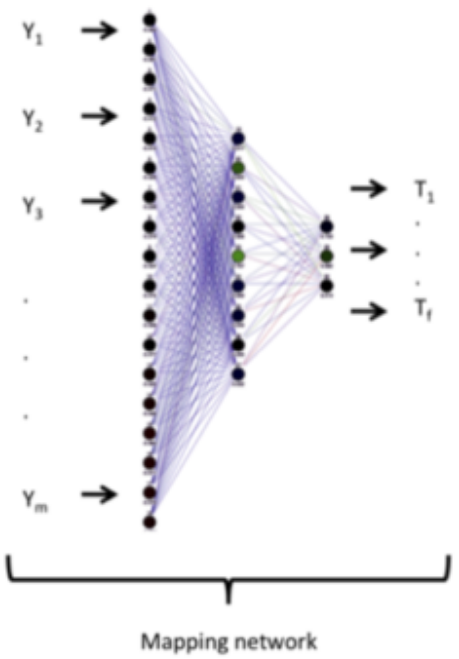

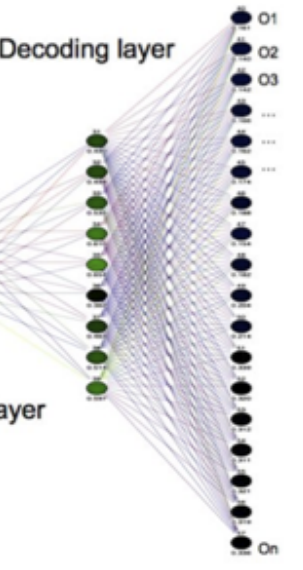

Output layer

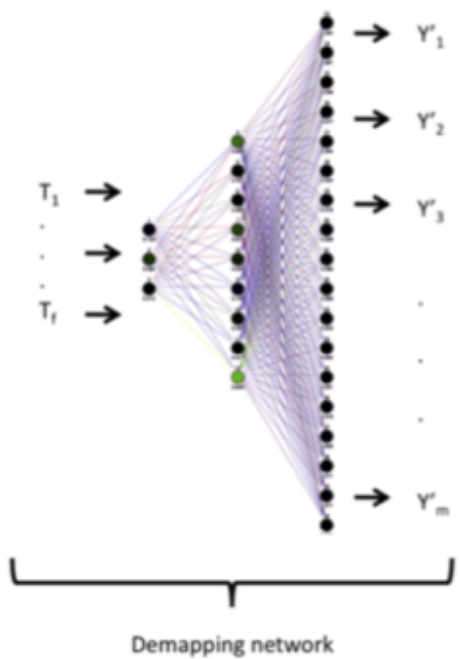

Fig. 1 General structure of the Autoassociative Neural Network. 


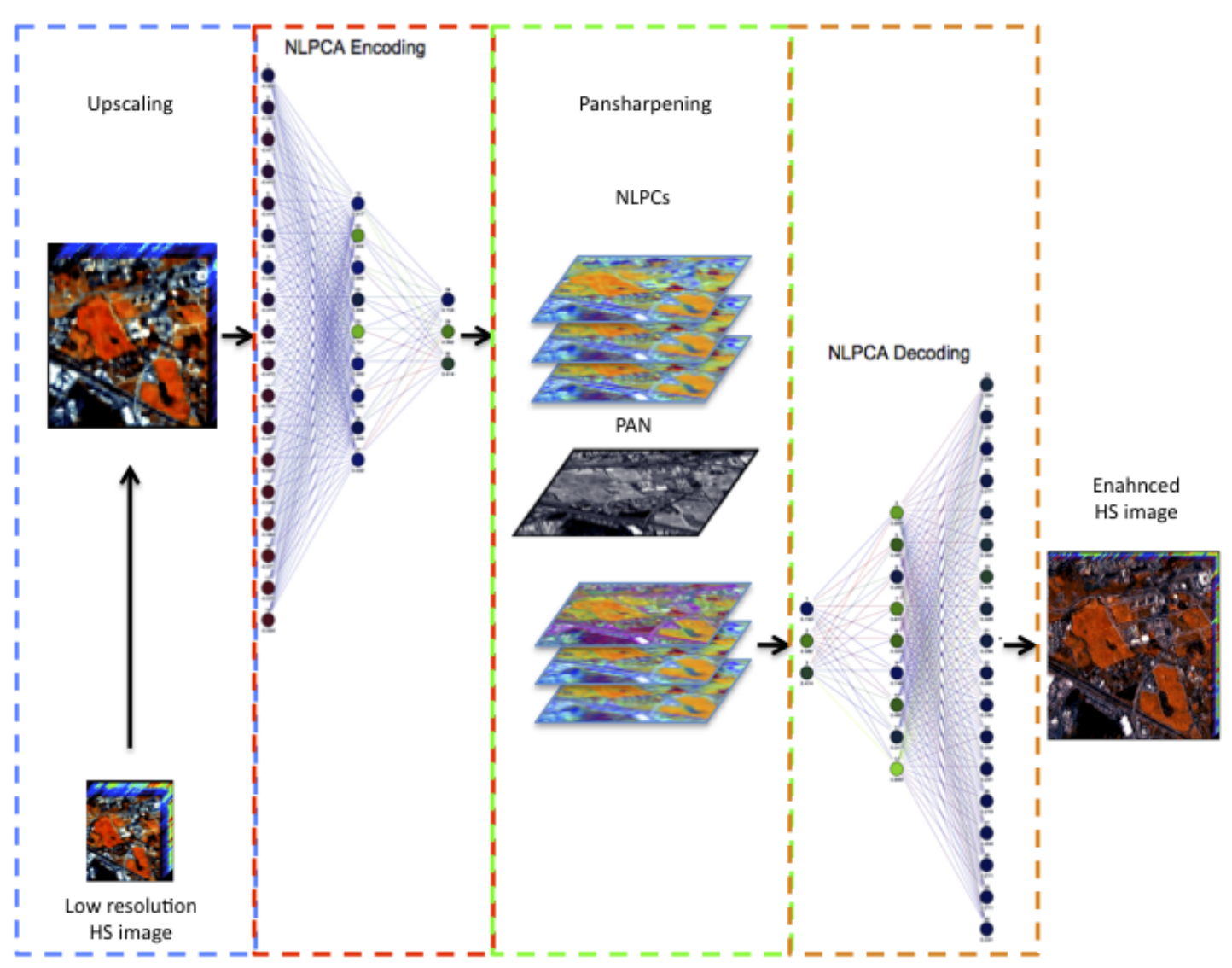

Fig. 2 Complete scheme of the proposed hybrid approach.
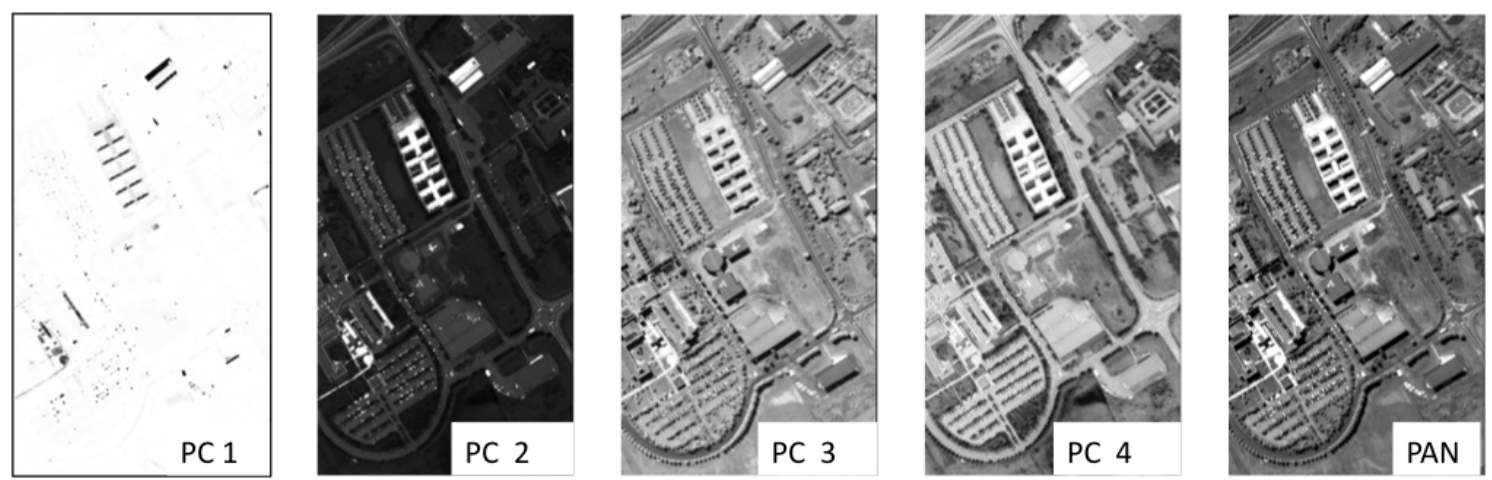

Fig. 3 ROSIS dataset: the 4 NLPCs obtained from the AANN and the original PAN image. 


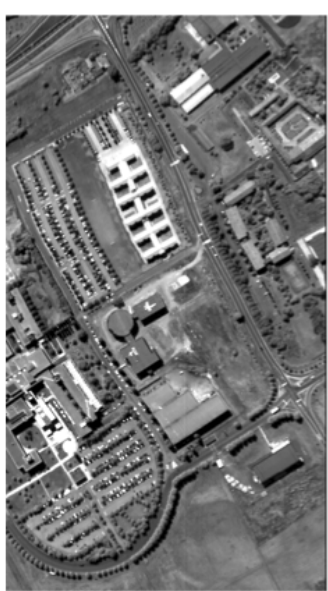

PAN

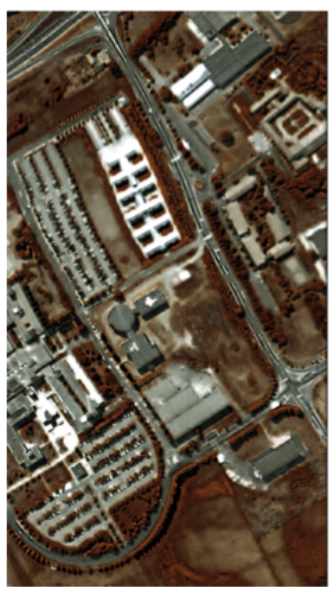

CBD

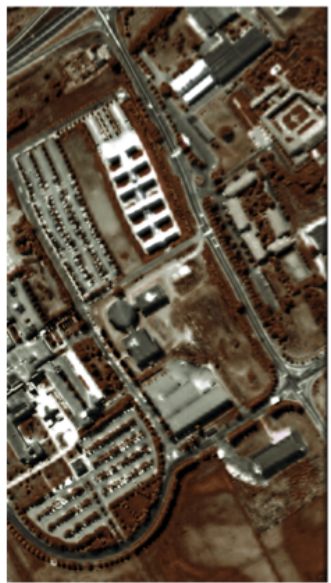

MTF-HPF

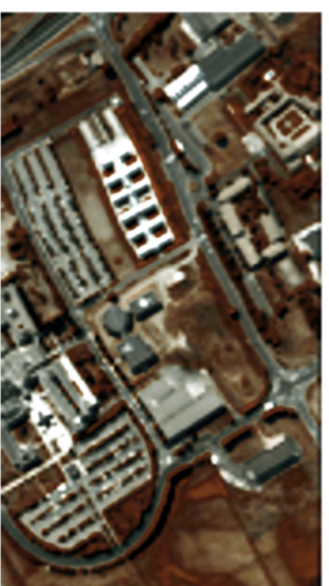

Low-Res HS

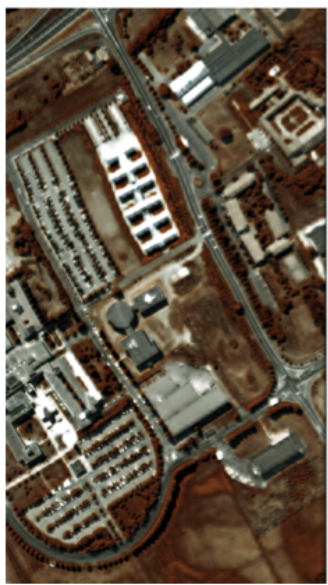

AWRGB

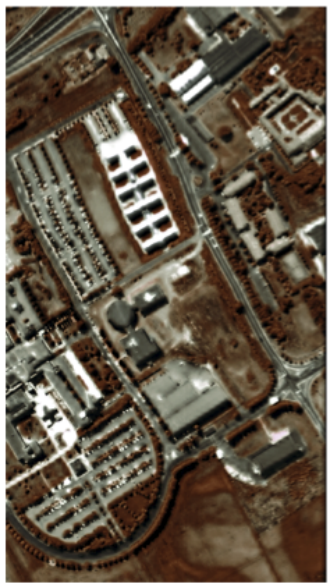

MTF-HPM

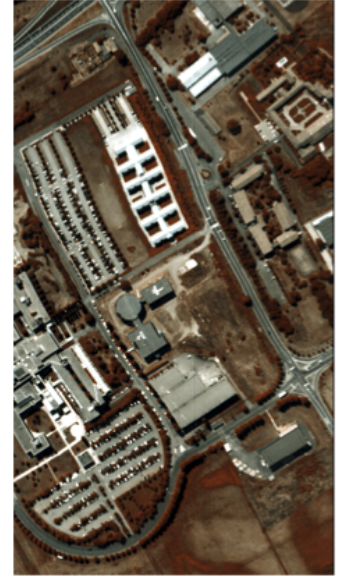

Reference

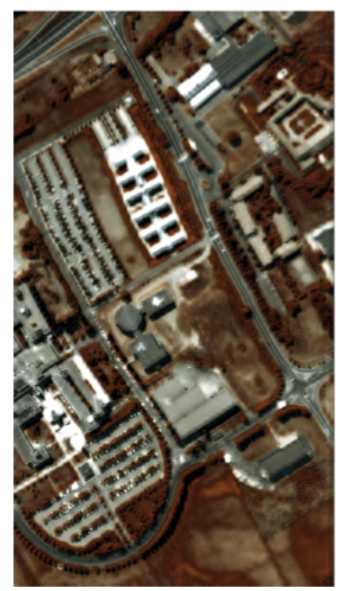

HPF

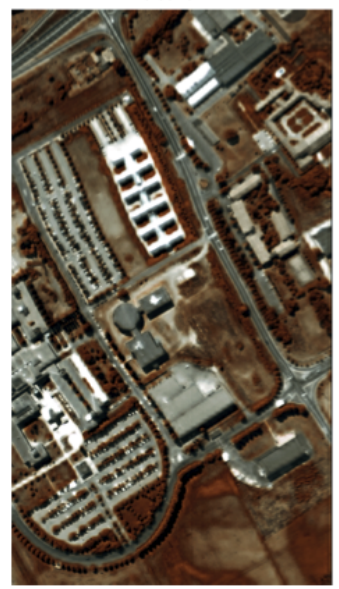

AWLP

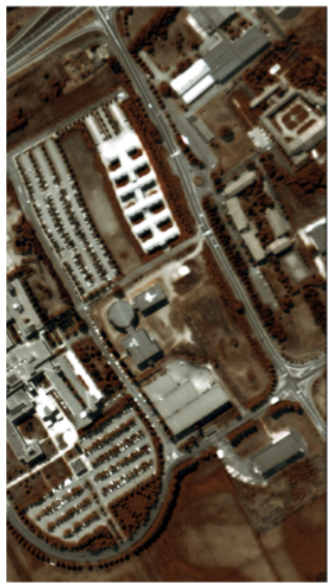

SUB

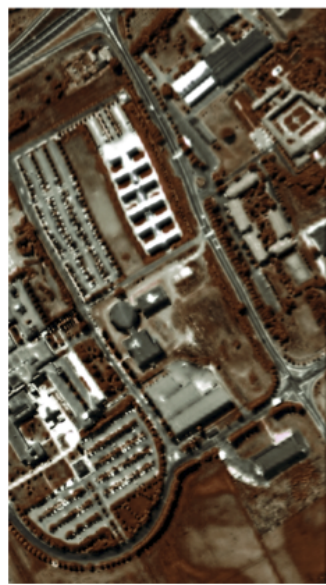

MF

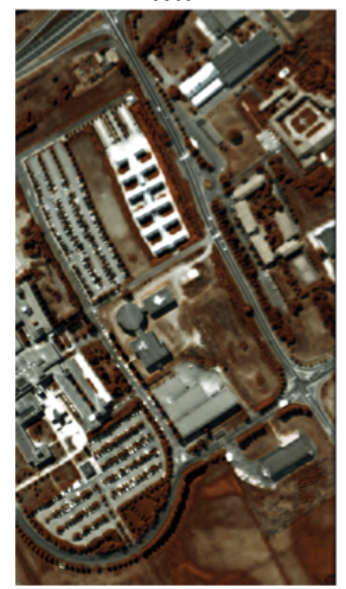

SFIM

Fig. 4 ROSIS dataset: RGB images obtained by combining bands 70, 50 and 20 of the enhanced images obtained with the different methods fusing NLPCs 2 and 3. 


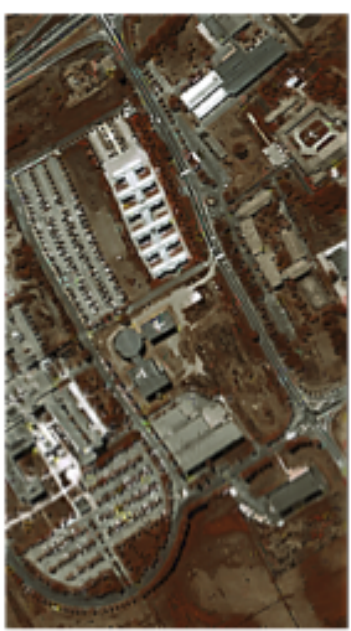

CBD

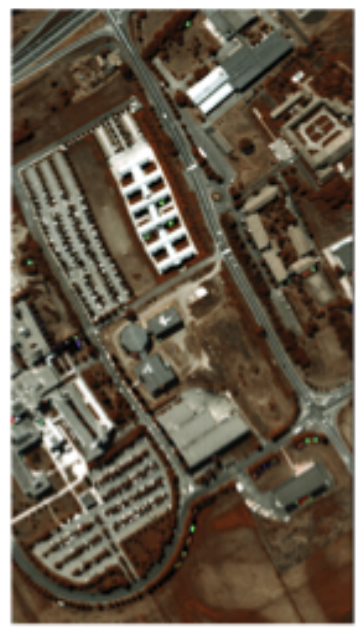

MF

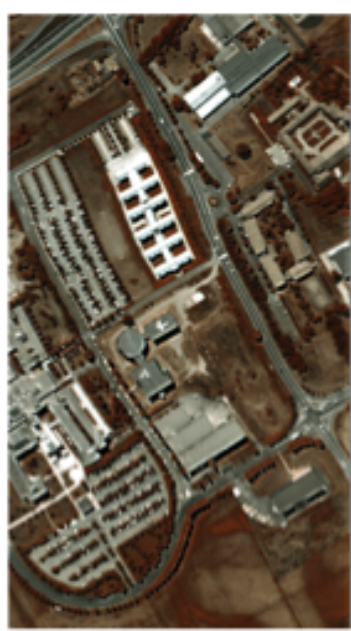

AWLP

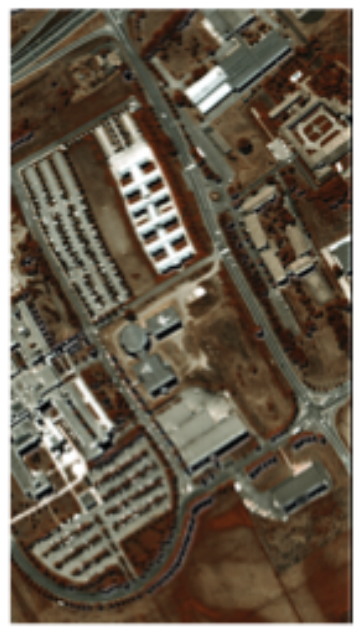

AWRGB

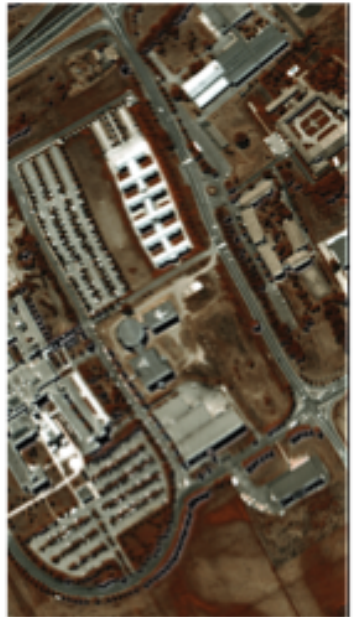

MTF-HPF

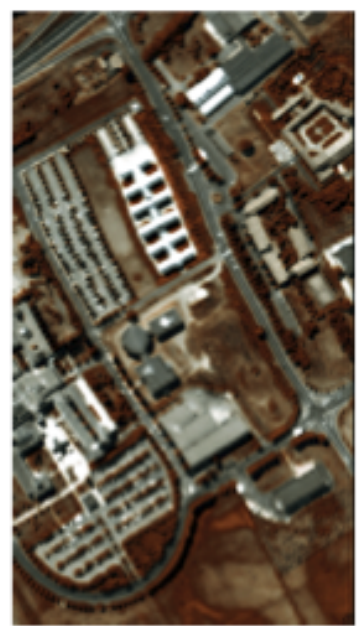

SFIM

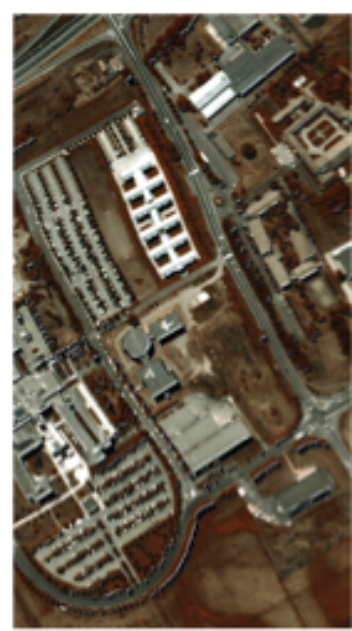

HPF

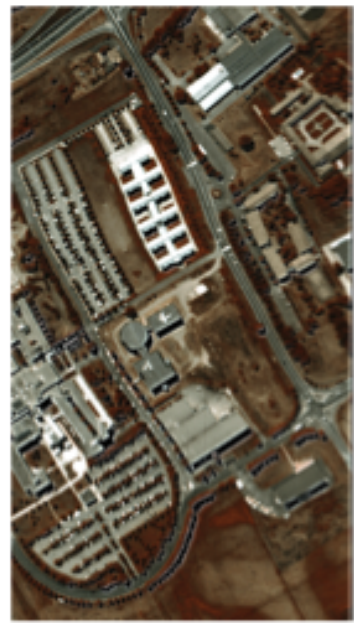

MTF-HPM

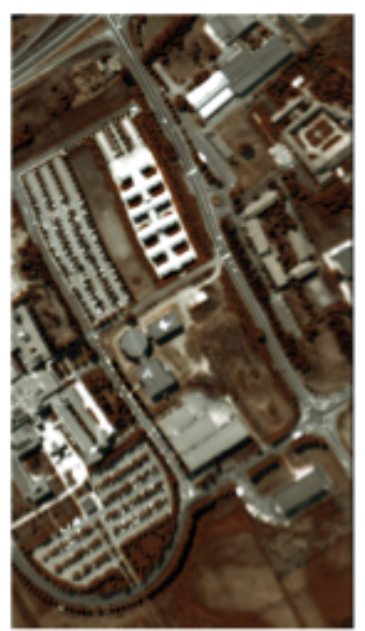

PCA

Fig. 5 ROSIS dataset: RGB images obtained by combining bands 70, 50 and 20 of the enhanced images obtained with the different methods applied to the original images. 

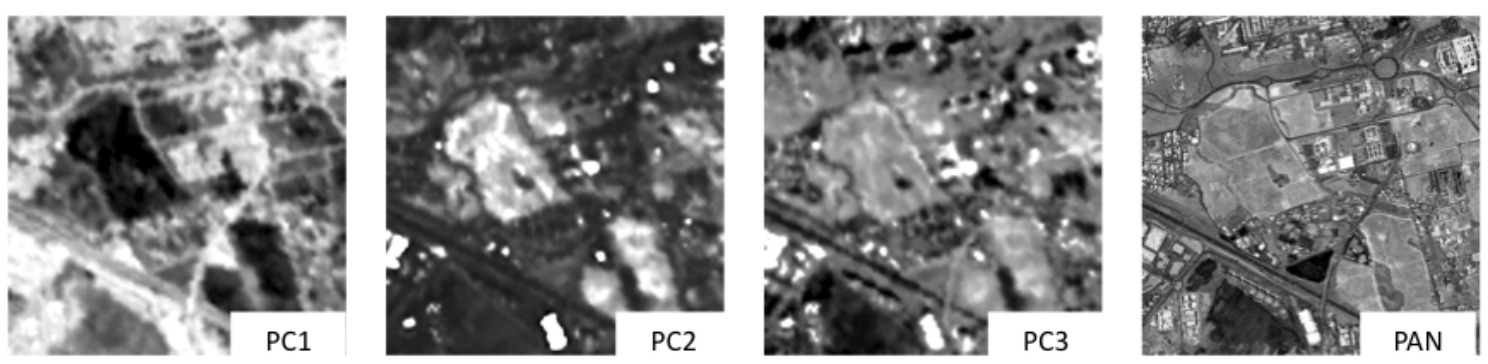

Fig. 6 CHRIS dataset: the 3 NLPCs and the original PAN image. 


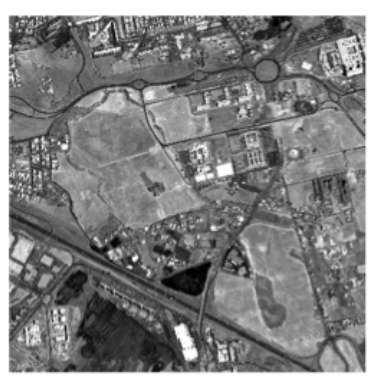

PAN

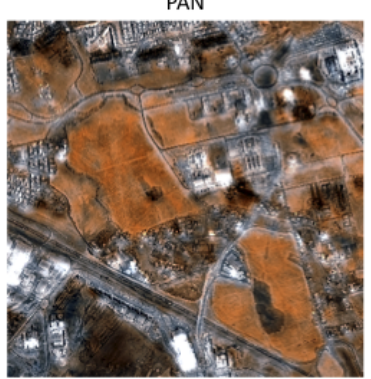

AWRGB

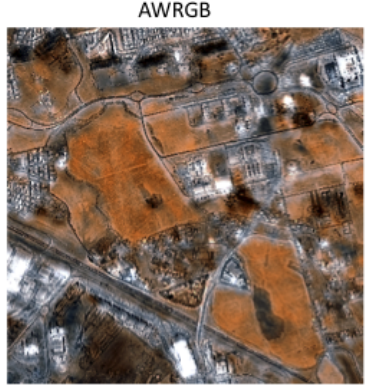

MTF-HPM

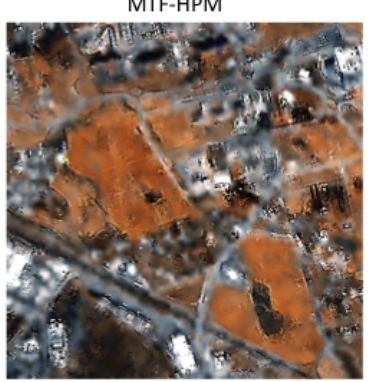

CBD - Original

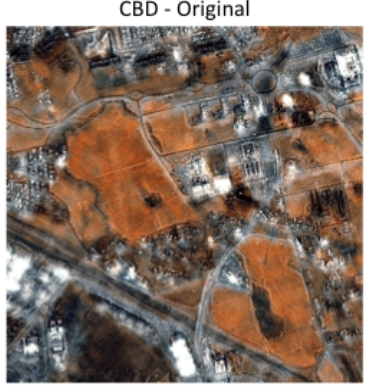

MTF-HPF - Original

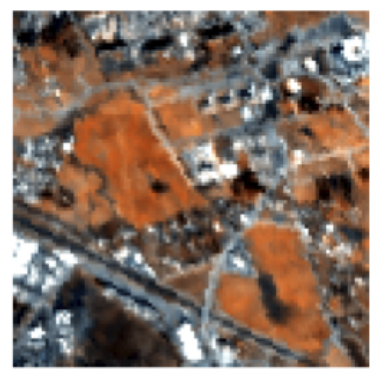

Low-Res HS

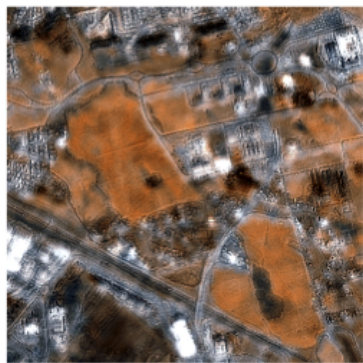

HPF

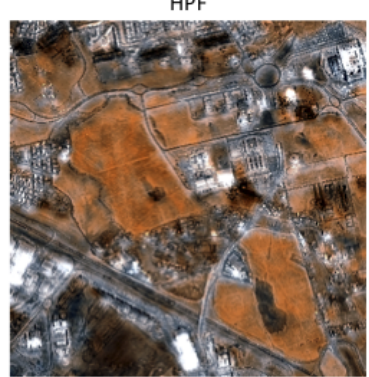

AWLP

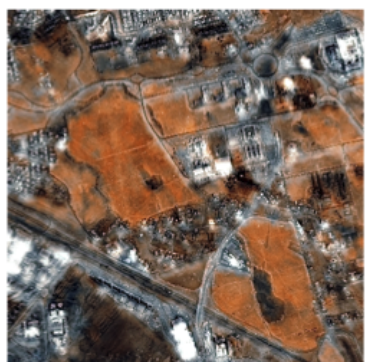

AWRGB - Original

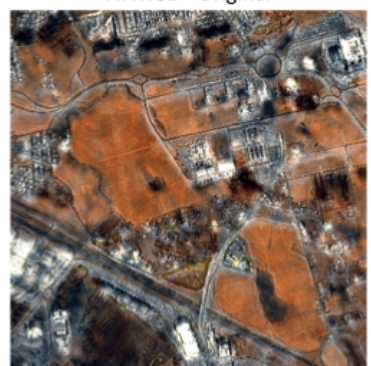

MTF-HPM - Original

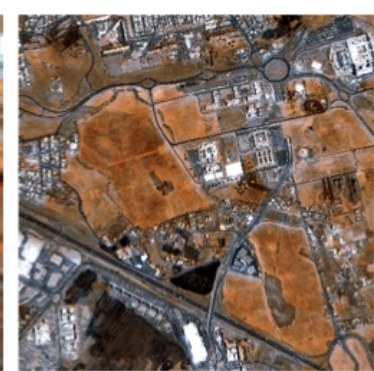

SUB

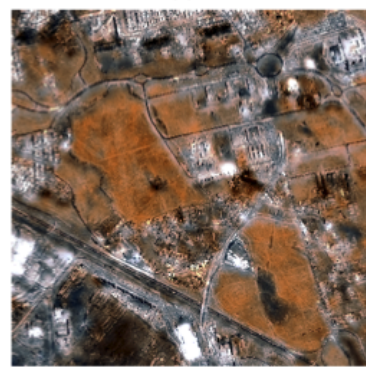

MF

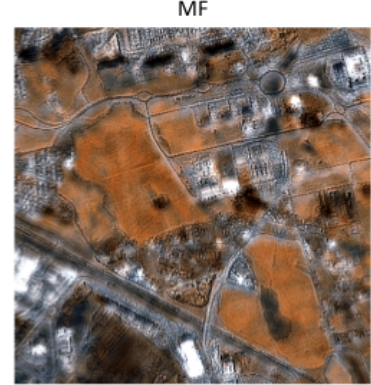

SFIM

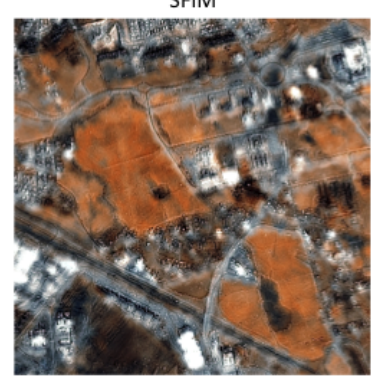

HPF - Original

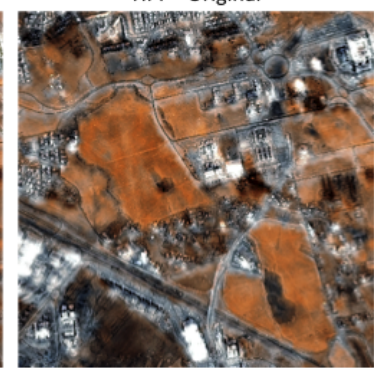

AWLP - Original

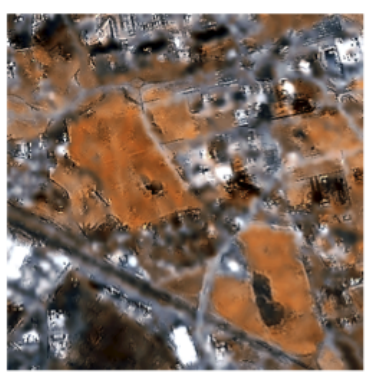

CBD

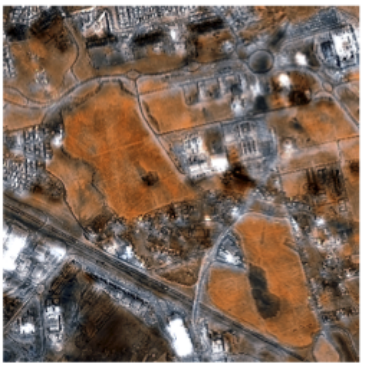

MTF-HPF

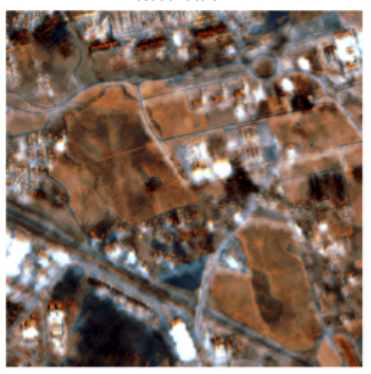

PCA - Original

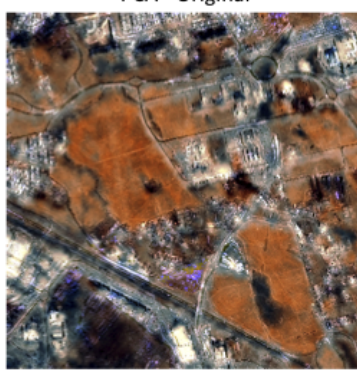

MF - Original

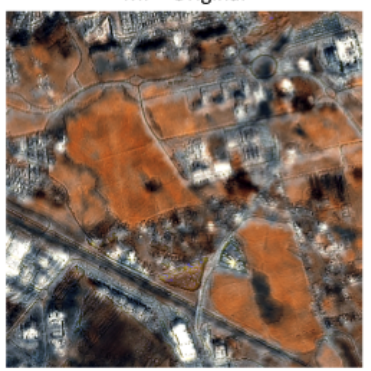

SFIM - Original

Fig. 7 CHRIS dataset: RGB images obtained by combining bands 11, 9 and 7 of the enhanced images obtained with the different methods. 


\section{References}

1. L. Alparone, L. Wald, J. Chanussot, C. Thomas, P. Gamba, and L. M. Bruce, "Comparison of pansharpening algorithms: Outcome of the 2006 grs-s data fusion contest," IEEE Trans. Geosci. Remote Sens., vol. 45, no. 10, pp. 3012-3021, 2007.

2. Thomas C., Ranchin T., Wald L., and Chanussot J., "Synthesis of multispectral images to high spatial resolution: A critical review of fusion methods based on remote sensing physics," IEEE Trans. Geosci. and Remote Sens., vol. 46, no. 5, pp. 1301-1312, May 2008.

3. Amro I., Mateos J., Vega M., Molina R., and Katsaggelos A.K., "A survey of classical methods and new trends in pansharpening of multispectral images," EURASIP Journal on Advances in Signal Processing, , no. 79, pp. 1-22, Sep 2011.

4. Burt P. and Adelson E., "The laplacian pyramid as a compact image code," IEEE Trans. Commun., vol. 31, no. 4, pp. 532-540, Apr 1983.

5. B. Aiazzi, L. Alparone, S. Baronti, and A. Garzelli, "Context-driven fusion of high spatial and spectral resolution images based on oversampled multiresolution analysis," IEEE Trans. Geosci. Remote Sens., vol. 40, pp. 2300-2312, 2002.

6. Aiazzi B., Alparone L., S. Baronti, A. Garzelli, and Selva M., "MTF-tailored multiscale fusion of high-resolution MS and Pan imagery," Photogramm. Eng. Remote Sens., vol. 72, no. 5, pp. 591-596, May 2006.

7. Shah V. P., Younan N. H., and King R. L., "An efficient pan-sharpening method via a combined adaptive PCA approach and contourlets," IEEE Trans. Geosci. Remote Sens., vol. 46, no. 5, pp. 1323-1335, May 2008.

8. Amolins K., Zhang Y., and Dare P., "Wavelet based image fusion techniques - an introduction, review and comparison," ISPRS Journal Photogramm, vol. 62, no. 4, pp. 249-263, Sep 2007.

9. Garguet-Duport N., Girel J., Chassery J.-M., and Patou G., "The use of multiresolution analysis and wavelets transform for merging spot panchromatic and multispectral image data," Photogram. Eng. Remote Sensing, vol. 62, no. 9, pp. 1057-1066, 1996.

10. T. Ranchin and L. Wald, "Fusion of high spatial and spectral resolution images: The arsis concept and its implementation," Photogramm. Eng. Remote Sens., vol. 66, no. 1, pp. 49-61, 2000.

11. J. Nunez, X. Otazu, O. Fors, A. Prades, V. Pala, and R. Arbiol, "Multiresolution-based image fusion with additive wavelet decomposition," IEEE Trans. Geosci. Remote Sens., vol. 37, no. 3, pp. 1204-1211, 1999.

12. Gonzalez-Audicana M., Otazu X., Fors O., and Seco A., "Comparison between mallat's and the a trous discrete wavelet transform based algorithms for the fusion of multispectral and panchromatic images," Int. J. Remote Sens., vol. 26, no. 3, pp. 595-614, 2005.

13. Haydn R., Dalke G. W., Henkel J., and Bare J. E., "Application of the IHS color transform to the processing of multisensor data and image enhancement," in Proc. Int. Symp. Remote Sens. Arid, Semi-Arid Lands, Cairo, Egypt, 1982, pp. 599-616.

14. Tu T. M., Huang P. S., Hung C. L., and Chang C.P., "A fast intensity-hue-saturation fusion technique with spectral adjustment for IKONOS imagery," IEEE Geosci. and Remote Sens. Letters, vol. 1, no. 4, pp. 309-312, Oct 2004.

15. T. M. Tu, S. C. Su, H. C. Shyu, and P. S. Huang, "A new look at ihs-like image fusion methods," Information Fusion, vol. 2, no. 3, pp. $177-186,2001$.

16. P. S. Chavez, S. C. Sides, and J. A. Anderson, "Comparison of three different methods to merge multiresolution and multispectral data: Landsat tm and spot panchromatic," Photogramm. Eng. Remote Sens., vol. 57, no. 3, pp. 295-303, 1991.

17. G. Licciardi, M. M. Khan, J. Chanussot, A. Montanvert, L. Condat, and C. Jutten, "Fusion of hyperspectral and panchromatic images using multiresolution analysis and nonlinear pca band reduction," EURASIP Journal of Advances in Signal Processing, vol. 2012:207, 2012.

18. M. A. Kramer, "Nonlinear principal component analysis using autoassociative neural networks," AIChE J., vol. 37, pp. 233-243, 1991.

19. M. M. Khan, J. Chanussot, L. Condat, and A. Montanvert, "Indusion: Fusion of multispectral and panchromatic images using the induction scaling technique," IEEE Geosci. Remote Sens. Lett., vol. 5, pp. 98-102, 2008. 
20. G. Licciardi, P. R. Marpu, J. Chanussot, and J. A. Benediktsson, "Linear versus nonlinear pca for the classification of hyperspectral data based on the extended morphological profiles," IEEE Geoscience and Remote Sensing Letters, vol. 9, pp. 447-451, 2012.

21. R. M. Cavalli, G. Licciardi, and J. Chanussot, "Detection of anomalies produced by buried archaeological structures using nonlinear principal component analysis applied to airborne hyperspectral image," IEEE Journal of Selected Topics in Applied Earth Observations and Remote Sensing, vol. PP, pp. 1-12, 2012.

22. L. K. Saul and S. T. Roweis, "Think globally, fit locally: Unsupervised learning of low dimensional manifolds," Journal of Machine Learning Research, vol. 4, no. 2, pp. 119-155, 2004.

23. J. Tenenbaum, V. de Silva, and J. Langford, "A global geometric framework for nonlinear dimensionality reduction," Science, vol. 290, no. 5500, pp. 2319-2323, 2000.

24. T. Kohonen, "Self-organizing maps," Springer, 3rd edition, 2001.

25. B. Scholkopf, A.J. Smola, and K.-R. Muller, "Nonlinear component analysis as a kernel eigenvalue problem," Neural Computation, vol. 10, pp. 1299-1319, 1998.

26. Cybenko G., "Approximations by superpositions of sigmoidal functions," Mathematics of Control, Signals, and Systems, vol. 2, no. 4, pp. 303-314, 1989.

27. C. Bishop, "Neural networks for pattern recognition," Oxford Univ. Press, London, U.K., 1995.

28. Cottrell G. W., P. Munro, and D. Zipser, "Learning internal representations from grayscale images: An example of extensional programming," Proc. Conf. of the Cognitive Sci. SOC., 1987.

29. G.A. Licciardi, M.M. Khan, and J. Chanussot, "Fusion of hyperspectral and panchromatic images: A hybrid use of indusion and nonlinear pca," in Image Processing (ICIP), 2012 19th IEEE International Conference on, 2012, pp. 2133-2136.

30. Soille P., "Morphological image analysis: principles and applications," Springer, 1999.

31. Lee J. and Lee C., "Fast and efficient panchromatic sharpening," Geoscience and Remote Sensing, IEEE Transactions on, vol. 48, no. 1, pp. 155-163, 2010.

32. Otazu X., Gonzalez-Audicana M., Fors O., and Nunez J., "Introduction of sensor spectral response into image fusion methods. application to wavelet-based methods," Geoscience and Remote Sensing, IEEE Transactions on, vol. 43, no. 10, pp. 2376-2385, 2005.

33. J. G. Liu, "Smoothing filter-based intensity modulation: A spectral preserve image fusion technique for improving spatial details," Int. J. Remote Sens., vol. 21, no. 18, pp. 3461-3472, 2000.

34. G. A. Licciardi, M. M. Khan, J. Chanussot, A. Montanvert, L. Condat, and C. Jutten, "Fusion of hyperspectral and panchromatic images using multiresolution analysis and nonlinear pca band reduction," IEEE Int. Geosci. Remote Sens. Symp. (IGARSS), 2011, pp. 1783-1786, 2011.

35. G. Licciardi, P.R. Marpu, J.A. Benediktsson, and J. Chanussot, "Extended morphological profiles using auto-associative neural networks for hyperspectral data classification," in Hyperspectral Image and Signal Processing: Evolution in Remote Sensing (WHISPERS), 20113 rd Workshop on, 2011, pp. 1-4.

36. L. Ward, "Data fusion: Definitions and architectures-fusion of images of different spatial resolutions.," Paris, France: ENSMP, 2002.

37. G. A. Licciardi and F. Del Frate, "Pixel unmixing in hyperspectral data by means of neural networks," IEEE Trans. Geosci. Remote Sens., vol. 49, no. 11, pp. 4163-4172, 2011. 